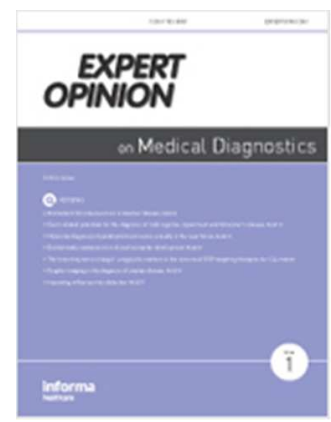

Please download and read the Referee Guidelines

Novel Diagnostic and Prognostic Biomarkers in Biliary Tract Cancer

\begin{tabular}{|r|l|}
\hline Journal: & Expert Opinion On Medical Diagnostics \\
\hline Manuscript ID: & EODG-2013-0029.R1 \\
\hline Manuscript Type: & Review \\
\hline Keywords: & $\begin{array}{l}\text { cholangiocarcinoma, biliary tract carcinoma, gallbladder cancer, } \\
\text { biomarkers, diagnosis, prognosis }\end{array}$ \\
\hline
\end{tabular}

SCHOLARONE $^{\mathrm{m}}$

Manuscripts

URL: http://mc.manuscriptcentral.com/eodg Email: anneke.prins@informa.com 


\title{
Novel Diagnostic and Prognostic Biomarkers in Biliary Tract Cancer
}

\begin{abstract}
Introduction: The worldwide incidence of biliary tract carcinoma (BTC; tumours of the bile ducts and gall-bladder) continues to rise with the only potentially curative treatment remaining surgical resection or transplantation, possible in only a minority of patients. Late presentation and a paucity of effective treatments mandate the development of techniques for early lesion detection.

Areas Covered: This article reviews currently available biomarkers for the diagnosis and prognosis of BTC, as well as recently published studies describing novel serum, bile and urinary biomarkers.

Expert Opinion: The incorporation of novel analysis techniques, such as digital image analysis and fluorescence in situ hybridization, into existing management algorithms may enhance the accuracy of brush cytology taken at the time of therapeutic endoscopy. However, a key goal is the discovery of reliable non-invasive biomarkers with high sensitivity and specificity. Recent advances in gene sequencing and expression, clonal evolution and tumour heterogeneity in other cancers should advance understanding of BTC tumour biology and facilitate biomarker discovery.
\end{abstract}

Keywords: Cholangiocarcinoma; Biliary Tract Cancer; Gallbladder Cancer; Biomarkers; Diagnosis; Prognosis 


\section{Introduction}

Biliary tract carcinoma (BTC) comprises tumours of the gallbladder and bile ducts, the commonest of which is cholangiocarcinoma $(\mathrm{CCa})$, an adenocarcinoma arising from the epithelium of the biliary tract and which is predominantly diagnosed in the $7^{\text {th }}$ decade of life [1]. CCa affects 1-2 per 100,000 in the UK population $[2,3]$ although the UK and worldwide incidence continues to rise with associated increasing mortality rates [3-6].

$\mathrm{CCa}$ can usually be considered to be sporadic but certain recognised predisposing factors have been identified, including primary sclerosing cholangitis (PSC; CCa occurs in up to $40 \%$ of PSC patients), prolonged or recurrent biliary infection, liver fluke infection (particularly in areas where Opisthorchis viverrini is endemic [7] cirrhosis, Caroli disease, the presence of choledochal cysts and hepatolithiasis, and carcinogen exposure (dioxins, nitrosamines, alcohol and thorotrast). BTC is also more prevalent in diabetics and smokers $[1,4]$. CCa may occur via an adenomadysplasia-carcinoma sequence, although CCa tumour biology remains poorly understood $[8,9]$. A number of mutations exist in tissue derived from CCa specimens, including abnormalities in known oncogenes and tumour suppressor genes; however, the frequency of such mutations is difficult to accurately estimate and this information, to date, remains clinically unusable [10-12]. Indeterminate biliary strictures present a diagnostic challenge to the investigating clinician. Many pathologies share clinical and radiological findings with the differentiation of BTC, PSC and autoimmune cholangiopathy remaining particularly difficult [13-15]. Endoscopic retrograde cholangiopancreatography (ERCP) can be utilized following cross-sectional radiological techniques for lesions assessment and biopsy with sensitivity for malignancy of 9-57\% [16-19]. Endoscopic ultrasound is a further well-established technique that can be used in conjunction with fine needle aspiration (FNA) for the visualization and sampling of gallbladder, hilar and extrahepatic lesions, as well as peri-hilar lymph nodes and vessels with a sensitivity for $\mathrm{CCa}$ of appproximately $75 \%$ [20]. Novel techniques such as peroral single operator cholangioscopy (e.g. Spyglass, Boston Scientific Corp, Natick, Massachusetts, USA) can have improved diagnostic accuracy as compared to standard ERCP [21] and has a specificity and sensitivity for visually directed biopsies of $98 \%$ and $49 \%$, respectively [22]. Further novel techniques, such as Methylene blue, which 
can successfully differentiate malignant lesions [23] and narrow-band imaging (NBI), which enhances the vascular pattern of the mucosal surface and can effectively differentiate tumour margins $[24,25]$, have also been utilised to augment the visualization of mucosa during cholangioscopy but are not routinely available. The majority of BTCs involve the main extrahepatic bile duct in the perihilar region $(60-70 \%)$, whereas the remainder tend to arise within the common bile duct (CBD) distal to the cystic duct. Only very infrequently do tumours arise in either smaller bile ducts within the liver parenchyma (intra-hepatic) or within the gall-bladder [26]. CCa cells tend to insidiously infiltrate and spread along the biliary tract, so that patients often have minimal clinical symptoms until late presentation, usually with cholestasis and evidence of locally advanced or metastatic disease on imaging [27-29]. The only chance for long-term survival remains radical resective surgery with negative pathological margins (or, in highly selected patients, liver transplantation). These strategies remain possible in only a minority of selected patients and result in postresection five year survival rates of up to $54 \%$, depending on site and severity of disease [30]. Non-surgical strategies currently provide only limited success in prolonging survival for those with advanced disease. Five-year survival rates in unresectable patients remain under $10 \%[30,31]$ with a median survival of usually less than 12 months following treatment with cisplatin- and gemcitabine-based chemotherapy regimens [32].

The lack of effective treatments for advanced disease mandates the development of techniques for early lesion detection. Whilst efforts to discover effective serum or bile biomarkers for early detection are ongoing, the relative rarity of the disease and the frequent presence of cholestasis and cholangitis which can confound bile assays, have so far limited discovery efforts.

This review assesses currently available biomarkers for the assessment of BTC, as well as reviewing recently published studies describing novel serum, bile and urinary biomarkers. A literature search was undertaken utilising PubMed and Embase search engines and the keywords cholangiocarcinoma; biliary tract cancer/carcinoma; gallbladder cancer/carcinoma; biomarkers; diagnosis; and prognosis entered in various combinations. 


\section{Serum Biomarkers}

Serum samples are easy to obtain and patient-acceptable, and therefore remain the most frequently utilized method of identifying biomarkers for $\mathrm{CCa}$.

\subsection{Established Serum Biomarkers}

\subsubsection{Carbohydrate Antigen 19-9 (CA19-9)}

CA19-9, or sialylated Lewis (a) antigen, remains the most widely used biomarker for the diagnosis of CCa. Despite this, it cannot be detected in $\sim 7 \%$ of the population who are Lewis (a) antigen negative (lacking the requisite fucosyltransferase for the production of CA19-9 [33, 34]) and is elevated in some other upper gastrointestinal malignancies (e.g. stomach and pancreatic adenocarcinoma), primary biliary cirrhosis, cholestasis, cholangitis and in smokers [35]. Indeed, $10 \%$ of patients under investigation for BTC (based upon radiological and clinical features) are found to have raised CA19-9 levels, but are subsequently found to have benign disease [36]. Serum bilirubin levels are also an independent predictor of serum CA19-9 levels: following intervention for cholestasis (with a subsequent drop in serum bilirubin), serum CA19-9 significantly decreases in tandem [36], thus limiting its specificity in patients presenting with cholestasis or cholangitis. However, if serum CA19-9 levels remain raised after intervention for cholestasis, this is strongly predictive (although not diagnostic) of malignant disease [37]. Depending upon the cut-off value used, CA19-9 has been shown to have a sensitivity range of $78-89 \%$ and a specificity range of $67-87 \%$ for the diagnosis of BTC [30, 36, 38, 39]. The use of higher serum CA19-9 cut-off levels decreases sensitivity, only allowing diagnosis of CCa to be made at an advanced stage of disease [33]. Further studies have attempted to use serum CA19-9 evaluation to differentiate between PSC-related and CCa-related strictures, and demonstrate wide-ranging but generally low sensitivity and specificity $[31,33,40]$. Further, the absolute value of CA19-9 may be affected by the specific assay used, with one study showing that different commercial assays deliver different results, potentially affecting the ability of CA19-9 to discriminate between malignant and benign disease [41]. One method for improving the diagnostic accuracy of CA19-9 may be to combine it with other biomarkers, such as bile-derived galectin-3-binding protein (LGALS3BP)/mac-2-binding protein levels, leading to significantly improved 
diagnostic accuracy over CA19-9 alone (area under ROC curve 0.75; $\mathrm{p}<0.001$ ) [42].

The sensitivity and specificity of CA19-9 also improves when combined with radiological imaging or endoscopic evaluation, particularly CT (computed tomography), MRI (magnetic resonance imaging), MRCP (magnetic resonance cholangiopancreatography) or ERCP (endoscopic retrograde cholangiopancreatography) [33]. At present, a combination of CA19-9 and MRI/MRCP or ultrasound represents the most effective, cost-efficient and acceptable technique for screening and follow-up of BTC.

\subsubsection{Carcinoembryonic Antigen (CEA)}

CEA is a glycosyl phosphatidyl inositol (GPI)-cell surface anchored glycoprotein that may be involved in cell adhesion. It is produced during foetal development, but its production tends to cease before birth and therefore levels in adults are generally low or non-existent, although they can be raised in smokers. It remains a useful biomarker in colorectal cancer, but is only raised in approximately $30 \%$ of patients with BTC $[39,43,44]$. On its own it has a limited role as a biomarker for BTC, but its clinical usefulness improves when used in combination with other markers [33].

\subsubsection{Carbohydrate Antigen 125 (CA125)}

CA125 is a protein encoded by the MUC16 gene and is a large membrane-associated glycoprotein with a single transmembrane domain. It is commonly employed for the detection of ovarian cancer, where it has a well-established role as a biomarker, but it is less useful for the detection of CCa where it is only elevated in $40-50 \%$ of CCa patients. When elevated, it may also indicate the presence of peritoneal involvement [6]. 


\subsection{Novel Serum Biomarkers}

Identification of novel serum markers to supplement or replace currently utilised biomarkers remains crucial. However, studies to date have not been able to demonstrate a suitable biomarker with high diagnostic accuracy. Some of the currently reported serum biomarkers for BTC are summarised below (see Table 1).

\subsubsection{Interleukin-6 (IL-6)}

IL-6 is a major inflammatory cytokine, whose high serum levels have been shown to correlate well with CCa tumour burden, both pre- and post-surgical resection [45]. However, high values can also be found in patients with associated inflammatory processes, such as cholestasis and cholangitis, as well as in hepatocellular carcinoma (HCC), metastatic liver lesions, and some benign liver pathologies [35]. Combining its use with CA19-9 (or other biomarkers) may improve its diagnostic accuracy. One group of authors demonstrated that at a cut-off level of $25.8 \mathrm{pg} / \mathrm{mL}$, IL-6 had a diagnostic sensitivity and specificity of $73 \%$ and $92 \%$ respectively (PPV and NPV $83 \%$ and $87 \%$, respectively), when utilized to differentiate between $26 \mathrm{CCa}, 26 \mathrm{HCC}$ and 23 healthy patients [46]. Serum levels were shown to correlate closely with tumour burden and also to decrease after treatment with photodynamic therapy. Similarly, the sensitivity and specificity for serum IL-6 (cut-off value $0.18 \mathrm{ng} / \mathrm{mL}$ ) in $45 \mathrm{CCa}$ patients was $71 \%$ and $90 \%$ for the differentiation of $\mathrm{CCa}$ and benign liver disease $(n=10)$, whereas the specificity for differentiating from 25 other hepatic tumours (15 HCC, 15 metastatic liver cancer) was only 26.7\% [47]. A combination of leucine-rich alpha-2 glycoprotein 1 (LRG-1), CA19-9 and IL-6 was also shown to be capable of discriminating $\mathrm{CCa}$ from benign biliary pathology with high diagnostic accuracy [48].

\subsubsection{Mucins}

Mucins are cell-specific, high molecular weight, heavily O-glycosylated glycoproteins expressed by ductal and glandular epithelia. They can be found in either transmembrane or secreted forms and have roles in epithelial protection, cellular adhesion and signal transduction. CCa neoplasms are commonly mucin-producing adenocarcinomas in which abnormal expression of various mucins, including mucin 1 (MUC1) and mucin5AC (MUC5AC), has been demonstrated and linked to poor outcomes. Importantly, MUC5AC expression has been confirmed in serum from $\mathrm{CCa}$ 
patients utilising various techniques including agarose gel electrophoresis, ELISA (enzyme-linked immunosorbent assay) and immunoblotting, but was not found in tissue derived from hepatocellular carcinomas (HCC). MUC5AC had a sensitivity and specificity of $71 \%$ and $90 \%$, respectively, (area under ROC curve 0.841 ) for the diagnosis of $\mathrm{CCa}$ (as compared to gastrointestinal cancer patients, benign hepatic pathology and healthy controls) and may therefore be able to differentiate between tumour types $[49,50]$. Further, those CCa patients with higher MUC5AC levels were shown to have poorer outcome (median survival 158 vs. 297 days).

A further study investigated serum MUC5AC and bile MUC4 using real-time PCR and western blotting in 72 patients with biliary obstruction (39 CCa, 7 PSC) [51]. Serum MUC5AC was again raised in 44\% of CCa patients (but not in patients with other malignancies), whilst bile MUC4 (collected at ERCP) was present in $27 \%$ of $\mathrm{CCa}$ cases, but not in the other malignancies. Further, those patients with either raised serum MUC5AC or bile MUC4 had significantly worse prognosis (median survival 6.8 vs. 17.6 months).

Serum MUC5AC was similarly measured in 179 CCa patients, where it was significantly associated with blood group A, tumours over $5 \mathrm{~cm}$ in size and advanced disease, as well as poorer prognosis, as compared with patients not expressing serum MUC5AC (median survival, 127 vs. 329 days; $\mathrm{P}<0.001$ ) [52]. Those patients expressing serum MUC5AC also had a 2.5 times increased risk of death $(\mathrm{p}<0.001)$. In further support for the potential role of mucins in CCa biology, MUC1 positivity was present in $65.8 \%$ of tumours obtained from 34 patients with intrahepatic $\mathrm{CCa}, 51$ with extrahepatic $\mathrm{CCa}, 11$ with gallbladder cancer and 14 patients with pancreatic adenocarcinoma, where it was associated with poor differentiation $(\mathrm{p}=0.002)$, T status $(p=0.003)$ and poor patient survival $(p=0.015)$ [53]. In the same study, MUC5AC was associated with advanced tumours $(\mathrm{p}=0.013)$ and MUC6 with well-differentiated CCa $(\mathrm{p}=0.006)$.

\subsubsection{CYFRA21-1}

CYFRA21-1 is a soluble fragment of cytokeratin 19, the intermediate filament protein that forms a crucial part of the epithelial cell cytoskeleton and is therefore constitutively expressed in many epithelial cells. It has been shown to be elevated in many different tumours including cervical, oesophageal and breast, but has a particular biomarker role in non small-cell lung carcinoma [54, 55]. 
Serum CYFRA21-1 levels can differentiate intrahepatic CCa from benign liver disease and $\mathrm{HCC}$, and have prognostic value [56]. In 55 patients who had undergone operative intervention for intrahepatic $\mathrm{CCa}$, as compared to 90 patients with benign liver pathology, the AUC was higher for CYFRA21-1 than CEA or CA19-9 (0.901, 0.779 and 0.794 , respectively). It had a sensitivity and specificity of $74.7 \%$ and $92.2 \%$ for $\mathrm{CCa}$ at a cut-off value of $2.7 \mathrm{ng} / \mathrm{mL}$. Furthermore, the 3-year recurrence-free survival rates were significantly worse in CCa patients with high serum CYFRA21-1 values $(25.0 \%$ vs. $76.2 \%)$ and CYFRA21-1 was found to be independently associated with recurrence and death following surgery on multivariate analysis [56, 57]. It has subsequently been postulated that raised CYFRA21-1 serum levels may represent a high burden of circulating tumour cells and that performing local operative resections in the presence of raised CYFRA21-1 may therefore prove futile [56].

However, another study group evaluated CYFRA21-1 in 66 patients with BTC (6 PSC-related), 39 patients with benign biliary disease and 19 patients with PSC to show that at a cut-off value of $1.5 \mathrm{ng} / \mathrm{mL}$, CYFRA21-1 had a sensitivity and specificity of $56 \%$ and $88 \%$, respectively, as compared to $79 \%$ and $78 \%$ for CA19-9 (cut-off value $37 \mathrm{IU} / \mathrm{mL}$ ), concluding that it was less useful than CA19-9 [58]. A higher cut-off value for CYFRA21-1 (3 ng/mL) improved specificity, but decreased sensitivity (97\% and 30\% respectively). Combining CYFRA21-1 and CA19-9 (cutoff values $1.5 \mathrm{ng} / \mathrm{mL}$ and $37 \mathrm{IU} / \mathrm{mL}$, respectively) led to a sensitivity and specificity of $45 \%$ and $96 \%$ and a high level of CYFRA $21-1(>3.0 \mathrm{ng} / \mathrm{mL})$ was a strong predictor of reduced median survival ( 2 vs. 10 months; $p<0.001$ ) [58].

\subsubsection{Trypsinogen}

Tumour-associated trypsinogens 1 and 2 are the precursor zymogen forms of the enzyme trypsin and are encoded by the same genes as pancreatic trypsinogen. A recent study demonstrated that serum trypsinogen-2 (PRSS2) was capable of differentiating between $\mathrm{CCa}$ and PSC in 84 patients referred for liver transplantation or other hepatic surgery more effectively than CA19-9, when assessed by timeresolved immunofluorometric assay with areas under the ROC curve (AUC) of 0.804 and 0.613 , respectively [59]. Trypsinogen-2 was also more effective at differentiating between PSC with and without CCa (AUC 0.759) and results were unaffected by the presence of other inflammatory conditions (e.g. inflammatory bowel disease) or high serum bilirubin levels. This potentially enhances the specificity of PRSS2 for the 
detection of $\mathrm{CCa}$, which frequently presents with cholangitis and cholestasis.

\subsubsection{Receptor Binding Cancer Antigen 1 (RCAS1)}

RCAS1 is a membrane protein that may participate in suppression of cell proliferation and induction of apoptotic cell death. Raised levels of serum RCAS1 were found in $73.9 \%$ of BTC patients (a higher percentage than CA19-9 or CEA), with a specificity of $96 \%$, and it was suggested that it may aid in monitoring recurrence [60-62]. Its levels were shown to reduce to within normal limits following surgical resection, were not affected by the presence of cholestasis and remained elevated in some patients where CA19-9 was normal, thereby providing a potential complementarity.

\subsubsection{Dickkopf-related protein 1 (DKK1)}

DKK1, a secreted protein with cysteine-rich regions involved in multiple cellular processes, via its inhibitory effect on the WNT signaling pathway, has been implicated in both metastatic spread and local invasion in several tumour types. Shi et al showed that DKK1 expression was raised in CCa cell lines, as well as sera and tissues from patients with $\mathrm{CCa}$ [63]. DKK 1 expression was also associated with elevated matrix metalloproteinase 9, VEGF-C and metastasis to hepatic hilar lymph nodes. Further, DKK1 was associated with poorer overall survival and time to recurrence on multivariate analysis, even in patients with low risk of recurrence $(\mathrm{p}<0.05)$. DKK1 down-regulation via siRNA decreased cell migration and invasiveness, as well as down-regulating MMP9 and VEGF-C expression.

\subsubsection{Other Biomarkers}

Proteomic analysis of serum samples is now readily available. Tolek et al analyzed serum proteomes from six CCa patients to identify $36 \mathrm{CCa}$-associated proteins, with $\alpha 1 \beta$-Glycoprotein (A1BG) and afamin (AFM) detected at consistently different levels in CCa patients, as compared to ten healthy controls [64]. A1BG and AFM were subsequently validated for their diagnostic and prognostic potential in $64 \mathrm{CCa}$ patients where the A1BG/AFM ratio was capable of diagnosing CCa with $84.4 \%$ sensitivity and $87.5 \%$ specificity. Furthermore, an elevated post-surgical ratio was associated with involved resection margins and poorer outcome.

Surface enhanced laser desorption/ionization (SELDI) analysis of serum from $56 \mathrm{CCa}$ patients, 49 patients with other hepatobiliary disease, 269 patients with other cancers 
and 53 healthy controls demonstrated three specific peaks that were significantly lower in CCa samples as compared to controls [65]. The peaks were all identified as variants of transthyretin (TTR or prealbumin), a largely hepatically-derived $55-\mathrm{kDa}$ protein which functions as a serum and cerebrospinal fluid carrier of thyroxine and thyroid hormones. TTR serum levels were subsequently assessed and confirmed to be decreased in CCa. Combining it with CA19-9 gave a sensitivity and specificity of $98.2 \%$ and $100.0 \%$ respectively, for differentiating CCa from benign disease.

However, TTR levels are also reduced in liver disease, malnutrition and acute inflammation, as well as other cancers such as ovarian, cervical and endometrial. Vascular endothelial growth factor (VEGF), a platelet-derived growth factor crucial for the stimulation of vasculogenesis and angiogenesis has been implicated in the proliferation and metastasis of a variety of solid tumours. Levels of serum VEGF were significantly higher in 29 patients with extrahepatic $\mathrm{CCa}$ and 19 patients with pancreatic cancer than in 25 patients with benign biliary pathologies (CBD stones, PSC and cholangitis) undergoing ERCP $(0.97 \mathrm{ng} / \mathrm{mL}(\mathrm{p}=0.0016)$ and $0.66 \mathrm{ng} / \mathrm{mL}$ $(p<0.001)$ vs. $0.28 \mathrm{ng} / \mathrm{mL}$, respectively) [66]. In contrast, bile concentrations of VEGF were similar between the three groups.

Li et al investigated levels of serum M2-PK, the dimeric form of the pyruvate kinase isoenzyme M2, which is responsible for energy production within the glycolytic pathway by catalyzing the dephosphorylation of phosphoenolpyruvate to pyruvate. M2-PK is the predominant isoform found within proliferating cells, such as fibroblasts, embryonic cells and stem cells and has been implicated in the metabolism of various tumour types. In 115 patients with $\mathrm{CCa}, 85$ with benign disease and 120 healthy blood donors, both M2-PK and CA19-9 were significantly higher in those with CCa and M2-PK was more sensitive and specific than CA19-9 for the diagnosis of malignancy ( $84.2 \%$ vs. $68.4 \%$ and $90 \%$ vs. $75 \%$, respectively) [67].

Jamnongkan et al investigated oxidized alpha-1 antitrypsin, an oxidative stress indicator for many pathological states, in opisthorchis-related $\mathrm{CCa}$ tissue and serum to demonstrate that patients with high tissue levels had a poorer prognosis [68]. Serum levels of oxidized alpha-1 antitrypsin were also significantly raised in patients with heavy infections, advanced periductal fibrosis and $\mathrm{CCa}$, as compared to healthy controls $(\mathrm{p}<0.001)$ and raised serum levels had an OR for CCa of 22.0, potentially representing a promising serum marker in areas where opisthorchis infection is 
1

2

3

4

5

6

7

8

9

10

11

12

13

14

15

16

17

18

19

20

21

22

23

24

25

26

27

28

29

30

31

32

33

34

35

36

37

38

39

40

41

42

43

44

45

46

47

48

49

50

51

52

53

54

55

56

57

58

59

60

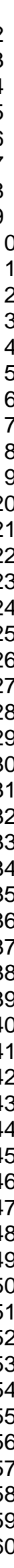 \\ Page 11 of $29 \quad$ Expert Opinion On Medical Diagnostics
}

\begin{abstract}
endemic. Further, SMAD7, involved in epithelial-mesenchymal transition has been
found to be elevated in CCa tissue and elevated expression associated with lymph

node metastsis, perineural invasion and survival [69].
\end{abstract}

(2)

(1)

(1)

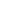

(1)

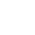

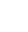

(1)

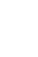

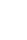




\section{Bile Biomarkers}

Bile provides a rich source of potential CCa biomarkers as it is more local to the tumour than serum. However, its collection remains an invasive process with significant potential complications and its use as a screening tool is therefore limited. The following reviews putative biomarkers identified within bile.

\subsection{Insulin-like Growth Factor-1 (IGF1)}

Human CCa tissues express IGF1, a 70 amino acid peptide hormone similar in molecular structure to insulin and which plays an important role in growth and anabolic processes, thereby also having a potentially crucial role in tumour development and proliferation [70]. IGF1 levels measured in bile from patients with biliary obstruction undergoing ERCP were up to 20 times higher in patients with extrahepatic $\mathrm{CCa}(\mathrm{n}=29)$ than those with pancreatic cancer $(\mathrm{n}=19)$ or benign biliary abnormalities (bile duct stones, PSC, cholangitis; $\mathrm{n}=25$ ) (mean, $84.6 \mathrm{nmol} / \mathrm{L}$ vs. 5.8 $\mathrm{nmol} / \mathrm{L}$ vs. $4.1 \mathrm{nmol} / \mathrm{L} ; \mathrm{p}<0.001$ with an area under the ROC curve of 1.0) [66]. Serum IGF-1 levels were also measured, but were similar between the three groups.

\subsection{Elastase}

Gene expression analysis, 2D electrophoretic analysis and mass spectrometry of fasting bile samples from patients undergoing ERCP or transhepatic catheterisation for biliary obstruction from malignant or benign causes (CCa or ductal stones ) demonstrated up-regulation of elastase mRNA, protein expression, and elastase activity in CCa compared to gallstone patients. The specific form was found to be elastase 3B, a pancreas-derived $29 \mathrm{kDa}$ serine protease, similar to classical pancreatic elastase 1 .

Biliary amylase levels were utilized to correct for the presence of pancreaticobiliary reflux and bile elastase/amylase ratios were significantly raised in CCa patients as compared to gallstone patients $(0.214+/-0.045$ vs. $0.023+/-0.005$, respectively; $\mathrm{p}<0.001$ ) with an area under the ROC curve of 0.877 . Moreover, the ratio had a sensitivity and specificity of $82 \%$ and $89 \%$ respectively, for differentiating malignant and benign causes of biliary obstruction (cut-off value 0.065 ) [71].

\subsection{Mini Chromosome Maintenance (MCM)}


MCM is the process of initiation and elongation of DNA replication and MCM replication proteins have been used as markers of dysplasia for the diagnosis of cervical and bladder cancer. Immunofluorometric analysis of bile sediment samples demonstrated that levels of MCM5, a protein which plays a role in transition from the G0 to G1/S phases of the cell cycle, were significantly more sensitive than brush cytology for the diagnosis of CCa (66\% vs. $20 \%$, respectively; $\mathrm{p}=0.004)$ with a comparable positive predicted value ( $97 \%$ vs. $100 \%$, respectively; $p=n s)$ in 60 patients with biliary strictures of indeterminate aetiology [72].

\subsection{Other Biomarkers}

Relative bile fibronectin concentrations (absolute fibronectin level divided by concentration of bile acids) measured in 62 gallstone patients with cholangitis, 5 with benign strictures and 28 with CCa were significantly different between the groups; and with a cut-off value of $40 \mathrm{ng} / \mu \mathrm{mol}$, its sensitivity and specificity for the diagnosis of CCa was 57\% and 79\%, respectively [73]. However, its increase in patients with cholangitis and cholestasis may limit its usefulness for the diagnosis of CCa. Shen et al recently compared bile protein expression profiles from patients with cholangitis and CCa, utilising a classic 2D gel and MS approach, identifying 97 differentially expressed protein spots derived from 49 different genes, 38 of which were up-regulated in CCa bile [74]. One of these proteins, spermatogenesis-associated protein 20 (SPATA20), was confirmed as being significantly upregulated in bile from CCa patients, and had a sensitivity and specificity for CCa diagnosis of $90.0 \%$ and $83.3 \%$ respectively. Alternate methods for bile sample preparation for proteomic analysis have also been employed, including prefractionation to enrich glycoproteins, leading to detection of CEA-related cell adhesion molecule 1, CA125, MUC2 and Galectin-3-binding protein (LGALS3BP), a highly glycosylated protein implicated in tumour development [57].

Dhar et al measured plasma and bile M2-PK levels in 88 patients with BTC, 79 with benign biliary diseases, and 17 healthy controls. Sensitivity (90.3\%) and specificity $(84.3 \%)$ of bile M2-PK for malignancy were significantly higher than those for plasma M2-PK and serum CA19-9 [75].

Alpini et al demonstrated that serotonin metabolism is dysregulated in $\mathrm{CCa}$ and that increased serotonin can be found in bile from CCa patients, where it may have 
implications for CCa cell growth [76]. The same group went on to show that mRNA and protein expression of the dopamine synthesis enzymes tyrosine hydroxylase and dopa decarboxylase were increased in CCa cell-lines and human biopsies [77]. Similarly, monoamine oxidase A (MAOA) was assessed in CCa and non-malignant controls to show that its expression correlated with differentiation, invasion and survival and that the MAOA promoter was hypermethylated upstream of the start codon in CCa samples and cell-lines. Further, IL-6 decreased MAOA expression [78] and may also upregulate progranulin expression and secretion via the ERK1/2/RSK1/C/EBP $\beta$ in CCa cell-lines and tissues where it increased CCa growth by an Akt-dependant mechanism [79]. 


\section{Urinary Biomarkers}

Urine also provides an easy and acceptable source for analysis of biomarkers, although its use in CCa remains limited to date. Metabolic profiling of small waste products in urine may, in the future, be able to identify certain patterns indicative of metabolic changes taking place in those with $\mathrm{CCa}$. Proteomic analysis of urine via capillary electrophoresis mass spectrometry recently assessed the distribution of 42 peptides (most being fragments of interstitial collagens of extra-renal origin) to correctly identify 35 of 42 CCa patients and 64 of 81 PSC and benign pathology patients with a sensitivity of $83 \%$ and a specificity of $79 \%$ [80]. Further, all of those with $\mathrm{CCa}$ in addition to PSC were correctly identified. 


\section{Conclusion}

Currently, the most widely utilized biomarkers for diagnosis of CCa include serum CA19-9, CA125 and CEA. However, CA19-9 levels are altered in a number of benign conditions, as well as in other upper abdominal malignant conditions. Further, approximately $7 \%$ of the population is Lewis negative and do not produce CA19-9, and it can also be low in up to $50 \%$ of patients with CCa. Similarly, CA125 and CEA are not consistently elevated in CCa patients and can be raised in a variety of both benign and malignant conditions. Thus, the need for better diagnostic and prognostic biomarkers in biliary tract carcinoma remains. A number of novel serum and biliary biomarkers have therefore been recently proposed. Models that combine multiple markers appear to significantly improve the diagnostic accuracy of existing markers such as CA19-9 and the identification of a suitable biomarker panel may provide a more viable means of success than any individual marker. However, further studies are required to replicate initial findings and investigate their applicability in a clinical setting.

The difficulty of obtaining bile for population screening and the lack of effective (both specific and sensitive) serum biomarkers means that suitable biomarkers for early detection and population screening are lacking. Alternate sources of biomarkers, including bile-rich gastric fluid via nasogastric aspiration or capsule endoscopy may ultimately prove to be a better technique. Further, a better understanding of tumour biology and the influence of various hormones and systemic factors may be necessary before effective biomarkers are found.

Possible future techniques for novel biomarker discovery include proteomic and gene expression profiling and the use of combinations of biomarkers to aid both diagnosis and choice of treatment regimen. However, proteomic analysis of bile and serum retains inherent problems in that they have very large dynamic ranges of expression with relatively few abundant proteins making up most of the protein content and contain contaminants that necessitate a significant degree of sample processing for their removal prior to analysis. 


\section{Expert Opinion}

Current biomarkers for the diagnosis of BTC have limited sensitivity and specificity, with particular difficulty in distinguishing PSC-related and malignant strictures, potentially contributing to late diagnosis with subsequent high mortality rates. The incorporation of novel analysis techniques, such as digital image analysis (DIA) and fluorescence in situ hybridization (FISH), into management algorithms may enhance the sensitivity of samples taken at ERCP, whilst maintaining the high specificity of brush cytology, which can be difficult to interpret, particularly in patients with PSC $[81,82]$. CCa has been associated with mutations in several oncogenes and up to $80 \%$ of tumour cells have been shown to exhibit chromosomal aneuploidy [83]. FISH and DIA can therefore be used to assess for the presence of these DNA abnormalities in brush cytology. They have been shown to improve the overall sensitivity for detecting $\mathrm{CCa}$ [82] and in PSC, where confirmation of $\mathrm{CCa}$ is particularly challenging, presence of polysomy is highly suggestive of CCa [84]. Similarly, techniques capable of assessing DNA-ploidy, such as flow cytometry, may improve the sensitivity of brush cytology [85]. However, such techniques require further validation and their routine use has only been adopted by a few centres [86]. Similarly, models that combine multiple biomarkers may improve the diagnostic accuracy of existing markers such as CA19-9 and the identification of a suitable biomarker panel that can be incorporated into treatment pathways may provide good diagnostic accuracy in the future.

A key goal in BTC is the discovery and validation of reliable, easily and consistently measurable biomarkers with good sensitivity and specificity. Statistical modelling of a biomarker signature in pancreatic cancer suggests that targeting $85 \%$ specificity would be acceptable in an enriched screening population with a cancer risk of $<1 \%$, results which may be applicable to specific at-risk groups in BTC (e.g. PSC) [87]. A better understanding of the underlying tumour biology of BTC would facilitate biomarker development. Various molecular processes have been described as being initiated during chronic cholestasis and associated local inflammation, such as that found during PSC, including release of various pro-inflammatory factors such as IL-6 and -8 , tumour necrosis factors, tumour growth factors and platelet-derived growth factors into the local environment, which may ultimately lead to DNA damage, apoptotic escape mechanisms and initiation of pro-oncological pathways, such as p53, 
p73, Smad4, K-ras, KIT, Bcl-2, survivin, cadherins and matrix metalloproteinase 7 [88-90]. Ultimately, such mechanisms may drive the development of BTC. Further, in patients with PSC, various cell-cycle and apoptotic control mechanisms, including those influenced by $\mathrm{p} 53$, Bcl-2, Bax and COX-2 pathways all played crucial roles in the pathogenesis of 128 BTCs (70 extrahepatic, 42 intrahepatic, 16 gallbladder) combined in a tissue microarray [91]. There was also differential expression of p16, p53 and Bcl-2 between intra- and extrahepatic CCa. p16 levels have subsequently been identified as having prognostic significance [91-94], as have the expression levels of epithelial-mesenchymal transition related genes, previously implicated in malignant invasion and poor prognosis [95] and HER3 of the receptor protein tyrosine kinase family [96]. Further, a specific isoform of p53 $(\Delta 133 \mathrm{p} 53)$ has now been identified as being associated with shorter overall survival than alternate p53 isoforms [97].

However, many of these pathways are crucial to multiple tumour types and little is known of the specific tumour biology underlying BTC development and progression. Comparison of intrahepatic CCA and $\mathrm{HCC}$ ( $\mathrm{n}=11$ and 24, respectively) tumours demonstrated specific chromosomal gains in $\mathrm{CCa}(20 \mathrm{q}, 5 \mathrm{p}, 7 \mathrm{q}$, and 13q) as compared to HCC (gains in1q and loss of 4q, 10q and 13q) [98]. Similarly, in 50 BTC tumours, gains in 1q, 8q and 20q and losses in 5q, 8q, 9p and 18q were consistently found in early and advanced tumours with loss of $9 \mathrm{p}$ being the commonest abnormality (78\% $\mathrm{T} 1 / 2$ and $68 \% \mathrm{~T} 3 / 4$ ) and the types of gains altered as per the presence or absence of lymph node (gains of 5p and 19q13 and losses of 6q14-q16) or distant metastatic disease (7p12-p14 $(\mathrm{P}<0.003)$, 7p21-pter $(\mathrm{P}<0.007)$ and 7q31 $(\mathrm{P}<0.01))$ [99]. Andresen et al utilized an epigenetic technique to identify that various proteins involved in signaling and oncogenic pathways, including cysteine dioxygenase type 1 (CDO1), serine/threonine-protein kinase DCLK1, secreted frizzled-related protein 1 (SFRP1) and zinc finger and SCAN domain containing 18 (ZSCAN18) had high methylation frequencies in $93 \mathrm{CCa}$ samples, as compared to being unmethylated in 43 control samples, with at least one of these four biomarkers being present in $87 \%$ of samples with a specificity of $100 \%$ [100]. microRNAs (miRNAs) are small non-coding RNAs (approximately 22 nucleotides) involved in regulating the expression of multiple genes and multiple cellular processes (including cell cycle, proliferation, apoptosis and migration) under both physiological and pathological conditions. This may also hold true for the 
development and progression of BTC [101, 102] and their expression may be epigenetically regulated by hormonal factors involved in tumour cell growth, such as IL-6 [103, 104]. Certain miRNAs, such as miR-421, miR-2 and miR-26a, are capable of acting in a pro-oncogenic fashion by down-regulating the effects of genes involved in tumour suppression such as FXR (farnesoid X receptor), arsenic resistance protein2, PTEN (phosphatase and tension homolog) and glycogen synthase kinase, and have been shown to be upregulated in CCa [105-108]. miRNA-25, which may help in regulating apoptotic pathways by protecting cholangiocytes from tumour necrosis factor and programmed cell death protein 4 , is over-expressed in CCa [109, 110]. Other miRNAs, such as miR-320, miR-204 and miR-29b which attenutate proapoptotic pathways, have been shown to be down-regulated [111, 112]. miRNA-21 and -200b are over-expressed in CCa and may also contribute to tumour progression by promoting resistance to chemotherapy [113].

Many solid tumours, including BTC, are characterized by RNA, DNA and chromosomal abnormalities and the initial work on gene expression, branched clonal evolution and tumour heterogeneity in other tumour types mandates further proteomic, metabolomic and genetic analysis of BTC tumours to aid understanding of tumour biology and facilitate biomarker develoment [114]. 


\section{Highlights Box}

1. Sensitivity and specificity of commonly utilized biomarkers remain inadequate for the diagnosis of patients with BTC, and the search for novel biomarkers remains crucial for the early detection of lesions.

2. Combinations of multiple biomarkers may improve the diagnostic accuracy of existing markers such as serum CA19-9.

3. Novel techniques, such as DIA and FISH, could be incorporated into existing management algorithms to enhance the accuracy of brush cytology.

4. The difficulty and invasiveness of obtaining bile for population screening mean that efforts should focus on the identification of an appropriate serum biomarker, unless alternate ways of obtaining bile for analysis (e.g. bile-rich gastric fluid via nasogastric aspiration or capsule endoscopy) prove effective.

5. Techniques for novel biomarker identification include proteomic and gene expression profiling; however, proteomic analysis of both bile and serum remains difficult due to the large dynamic ranges of expression with relatively few abundant proteins making up most of the protein content.

6. Improved understanding of tumour biology may allow targeted analysis of serum and bile samples for effective biomarkers. 


\section{Annotated Bibliography}

1. West, J., Wood, H., Logan, R.F., et al., Trends in the incidence of primary liver and biliary tract cancers in England and Wales 1971-2001. Br J Cancer, 2006. 94: p. 1751-8

2. Patel, T., Increasing incidence and mortality of primary intrahepatic cholangiocarcinoma in the United States. Hepatology, 2001. 33: p. 1353-7

3. Taylor-Robinson, S.D., Toledano, M.B., Arora, S., et al., Increase in mortality rates from intrahepatic cholangiocarcinoma in England and Wales 19681998. Gut, 2001. 48: p. 816-20

4. Endo, I., Gonen, M., Yopp, A.C., et al., Intrahepatic cholangiocarcinoma: rising frequency, improved survival, and determinants of outcome after resection. Ann Surg, 2008. 248: p. 84-96

5. Khan, S.A,, Miras, A., Pelling, M., et al., Cholangiocarcinoma and its management. Gut, 2007. 56: p. 1755-6

6. Khan, S.A., Davidson, B.R., Goldin, R., et al., Guidelines for the diagnosis and treatment of cholangiocarcinoma: consensus document. Gut, 2002. 51 Suppl 6: p. VI1-9

7. Saengsawang, P., S. Promthet, and P. Bradshaw, Infection with Opisthorchis viverrini and Use of Praziquantel among a Working-age Population in Northeast Thailand. Asian Pac J Cancer Prev. 14: p. 2963-6

8. $\quad$ Fleming, K.A., Boberg, K.M., Glaumann, H., et al., Biliary dysplasia as a marker of cholangiocarcinoma in primary sclerosing cholangitis. J Hepatol, 2001. 34: p. 360-5

9. Lewis, J.T., Talwalkar, J.A., Rosen, C.B., et al., Precancerous bile duct pathology in end-stage primary sclerosing cholangitis, with and without cholangiocarcinoma. Am J Surg Pathol, 2010. 34: p. 27-34

10. Enjoji, M., M. Nakamuta, and H. Nawata, Oncogenes in tumors of gallbladder and biliary tract. Fukuoka Igaku Zasshi, 2004. 95: p. 31-5

11. Klump, B., Hsieh, C.J., Dette, S., et al., Promoter methylation of INK4a/ARF as detected in bile-significance for the differential diagnosis in biliary disease. Clin Cancer Res, 2003. 9: p. 1773-8

12. Morales, C.P., Burdick, J.S., Saboorian, M.H., et al., In situ hybridization for telomerase RNA in routine cytologic brushings for the diagnosis of pancreaticobiliary malignancies. Gastrointest Endosc, 1998. 48: p. 402-5

13. Saluja, S.S., Sharma,R., Pal S, Sahni P, Chattopadhyay TK., Differentiation between benign and malignant hilar obstructions using labaratory and radiological investigations: a prospective study. HPB (Oxford), 2007. 9: p. 373-82

14. Fernandez- Esparrach G, G.A., Sanchez, M., Pagés, M., et al, Comparison of endoscopic ultrasonography and magnetic resonance cholangiopancreatography in the diagnosis of pancreatobiliary diseases: a prospective study. American Journal of Gastroenterology, 2007. 102: p. 16329

15. Sai JK, S.M., Kubokawa, Y., Watanabe, S., et al., Early detection of extrhepatic bile-duct carcinomas in the nonicteric stage by using MRCP followed by EUS. Gastrointestinal Endoscopy, 2009. 70: p. 29-36

16. de Bellis M, S.S., Fofel, E.L., Cramer, H., et al, Tissue sampling at ERCP in suspected malignant biliary strictures (Part 2). Gastrointestinal Endoscopy, 2002. 56: p. $720-730$ 
17. Baron, T.H., Harewood.G.C., Rumalla, A., et al, A prospective comparison of digital image analysis and routine cytology for the identification of malignancy in biliary tract strictures. Clin Gastroenterol Hepatol, 2004. 2: p. 214-219

18. Harewood, G.C., Baron,T.H., Stadheim, L.M., et al, Prospective, blinded assessment of factors influencing the accuracy of biliary cytology interpretation. American Journal of Gastroenterology, 2004. 99: p. 1464-1469

19. Moreno Luna, L.E., Kipp, B., Halling, K.C., et al, Advanced cytologic techniques for the detection of malignant pancreobiliary strictures. Gasroenterology, 2006. 131: p. 1064-1072

20. Mohamadnejad, M., DeWitt, J.M., Sherman, S., Role of EUS for preoperative evaluation of cholangiocarcinoma: a large single-center experience. Gastrointestinal Endoscopy, 2011. 73(1): p. 71-8

21. Tischendorf, J.J., Krüger, M., Trautwein, C., et al, Cholangioscopic characterization of dominant bile duct stenoses in patients with primary sclerosing cholanditis. Endoscopy, 2006. 38: p. 665-669

22. Chen, Y.K., Parsi, M.A., Binmoeller, K.F. et al, Single-operator cholangioscopy in patients requiring evaluation of bile duct disease or therapy of biliary stones (with video). Gastrointestinal Endoscopy, 2011. 74: p. 804-814

23. Hoffman, A., Kiesslich, R., Bittinger, F., et al, Methylene blue-aided cholangioscopy in patients with biliary strictures: feasibility and outcome analysis. . Endoscopy, 2008. 40: p. 563-571

24. Itoi, T., Sofuni, A., Itokawa, F., et al, Peroral cholangioscopic diagnosis of biliary-tract diseases by using narrow-band imaging (with videos). Gastrointestinal Endoscopy, 2007. 19

25. Lu, X.L., Itoi, T., Kubota, K., Cholangioscopy by using narrow-band imaging and transpapillary radiotherapy for mucin-producing bile duct tumour. Clin Gastroenterol Hepatol, 2009. 7: p. e34-e35

26. Nakeeb, A., Pitt, H.A., Sohn, T.A., et al., Cholangiocarcinoma. A spectrum of intrahepatic, perihilar, and distal tumors. Ann Surg, 1996. 224: p. 463-73; discussion 473-5

27. Blechacz, B.R., W. Sanchez, and G.J. Gores, A conceptual proposal for staging ductal cholangiocarcinoma. Curr Opin Gastroenterol, 2009. 25: p. 238-9

28. Matull, W.R., S.A. Khan, and S.P. Pereira, Re: Impact of classification of hilar cholangiocarcinomas (Klatskin tumors) on incidence of intra- and extrahepatic cholangiocarcinoma in the United States. J Natl Cancer Inst, 2007. 99: p. 407; author reply 407-8

29. Anderson, C.D., Pinson, C.W., Berlin, J., et al., Diagnosis and treatment of cholangiocarcinoma. Oncologist, 2004. 9: p. 43-57

30. Khan, S.A., Thomas, H.C., Davidson, B.R., et al., Cholangiocarcinoma. Lancet, 2005. 366: p. 1303-14.

31. Reddy, S.B. and T. Patel, Current approaches to the diagnosis and treatment of cholangiocarcinoma. Curr Gastroenterol Rep, 2006. 8: p. 30-7

32. Valle, J., Wasan, H., Palmer, D.H., et al., Cisplatin plus gemcitabine versus gemcitabine for biliary tract cancer. N Engl J Med. 362: p. 1273-81

33. Nehls, O., M. Gregor, and B. Klump, Serum and bile markers for cholangiocarcinoma. Semin Liver Dis, 2004. 24: p. 139-54 
34. Locker, G.Y., Hamilton, S., Harris, J., et al., ASCO 2006 update of recommendations for the use of tumor markers in gastrointestinal cancer. J Clin Oncol, 2006. 24: p. 5313-27

35. पDBonney, G.K., Craven, R.A., Prasad, R., et al., Circulating markers of biliary malignancy: opportunities in proteomics? Lancet Oncol, 2008. 9: p. $149-58$

An excellent review describing the use of proteomic-based approaches for thr assessment of biomarkers in biliary malignancy. Proteomics complements genomic studies and examines functional end-units quantitatively and qualitatively, including post-translational modifications which might vary with disease and might have key roles in protein function or localisation.

36. Kim, H.J., Kim, M.H., Myung, S.J., et al., A new strategy for the application of CA19-9 in the differentiation of pancreaticobiliary cancer: analysis using a receiver operating characteristic curve. Am J Gastroenterol, 1999. 94: p. 1941-6

37. Ventrucci, M., Pozzato, P., Cipolla, A., et al., Persistent elevation of serum CA 19-9 with no evidence of malignant disease. Dig Liver Dis, 2009. 41: p. 35763

38. Shaib, Y.H., Davila, J.A., McGlynn, K., et al., Rising incidence of intrahepatic cholangiocarcinoma in the United States: a true increase? J Hepatol, 2004. 40: p. 472-7

39. Abi-Rached, B. and A.I. Neugut, Diagnostic and management issues in gallbladder carcinoma. Oncology (Williston Park), 1995. 9: p. 19-24; discussion 24, 27, 30

40. Sinakos, E., Saenger, A.K., Keach, J., Many patients with primary sclerosing cholangitis and increased serum levels of carbohydrate antigen 19-9 do not have cholangiocarcinoma. Clin Gastroenterol Hepatol. 9: p. 434-9 e1

41. Hotakainen, K., Tanner, P., Alfthan, H., Comparison of three immunoassays for CA 19-9. Clin Chim Acta, 2009. 400: p. 123-7

42. Koopmann, J., Thuluvath, P.J., Zahurak, M.L., et al., Mac-2-binding protein is a diagnostic marker for biliary tract carcinoma. Cancer, 2004. 101: p. 160915

43. Lazaridis, K.N. and G.J. Gores, Primary sclerosing cholangitis and cholangiocarcinoma. Semin Liver Dis, 2006. 26: p. 42-51

44. LaRusso, N.F., Shneider, B.L., Black, D., Primary sclerosing cholangitis: summary of a workshop. Hepatology, 2006. 44: p. 746-64

45. Goydos, J.S., Brumfield, A.M., Frezza, E., et al., Marked elevation of serum interleukin-6 in patients with cholangiocarcinoma: validation of utility as a clinical marker. Ann Surg, 1998. 227: p. 398-404

46. Cheon, Y.K., Cho, Y.D., Moon, J.H., et al., Diagnostic utility of interleukin-6 (IL-6) for primary bile duct cancer and changes in serum IL-6 levels following photodynamic therapy. Am J Gastroenterol, 2007. 102: p. 2164-70

47. Tangkijvanich, P., Thong-ngam, .D, Theamboonlers, A., et al., Diagnostic role of serum interleukin 6 and CA 19-9 in patients with cholangiocarcinoma. Hepatogastroenterology, 2004. 51: p. 15-9

48. Sandanayake, N.S., Sinclair, J., Andreola, F., et al., A combination of serum leucine-rich alpha-2-glycoprotein 1, CA19-9 and interleukin-6 differentiate biliary tract cancer from benign biliary strictures. Br J Cancer. 105: p. 1370-8 
49. Bamrungphon, W., Prempracha, N., Bunchu, N., et al., A new mucin antibody/enzyme-linked lectin-sandwich assay of serum MUC5AC mucin for the diagnosis of cholangiocarcinoma. Cancer Lett, 2007. 247: p. 301-8

50. Wongkham, S., Sheehan, J.K., Boonla, C., et al., Serum MUC5AC mucin as a potential marker for cholangiocarcinoma. Cancer Lett, 2003. 195: p. 93-9

51. QDMatull, W.R., Andreola, F., Loh, A., et al., MUC4 and MUC5AC are highly specific tumour-associated mucins in biliary tract cancer. Br J Cancer, 2008. 98: p. $1675-81$

The authors assessed MUC4 and MUC5AC expression in prospectively collected bile and serum specimens from 72 patients with biliary obstruction and 79 archived biliary tissues. In bile, MUC4 protein was detected in $27 \%$ of BTC and $29 \%$ of primary sclerosing cholangitis (PSC) cases, but not in other benign and malignant biliary diseases $(\mathrm{P}<0.01$ and $\mathrm{P}=0.06)$. qPCR revealed a 1.9 -fold increased MUC4 mRNA expression in BTC patients' bile compared with benign disease. In serum, MUC5AC was found exclusively in BTC and PSC sera (44\% and 13\%, respectively; $\mathrm{P}<0.001$ for BTC vs non-BTC) and correlated negatively with BTC survival.

52. Boonla, C., Wongkham, S., Sheehan, J.K., et al., Prognostic value of serum MUC5AC mucin in patients with cholangiocarcinoma. Cancer, 2003. 98: p. 1438-43

53. Park, S.Y., Roh, S.J., Kim, Y.N., et al., Expression of MUC1, MUC2, MUC5AC and MUC6 in cholangiocarcinoma: prognostic impact. Oncol Rep, 2009. 22: p. 649-57

54. Yi, Y., Li, B., Wang, Z., et al., CYFRA21-1 and CEA are useful markers for predicting the sensitivity to chemoradiotherapy of esophageal squamous cell carcinoma. Biomarkers, 2009. 14: p. 480-5

55. Mizuguchi, S., Nishiyama, N., Iwata, T., et al., Serum Sialyl Lewis $x$ and cytokeratin 19 fragment as predictive factors for recurrence in patients with stage I non-small cell lung cancer. Lung Cancer, 2007. 58: p. 369-75

56. Uenishi, T., Yamazaki, O., Tanaka, H., et al., Serum cytokeratin 19 fragment (CYFRA21-1) as a prognostic factor in intrahepatic cholangiocarcinoma. Ann Surg Oncol, 2008. 15: p. 583-9

57. Kashihara, T., Ohki, A., Kobayashi, T., et al., Intrahepatic cholangiocarcinoma with increased serum CYFRA 21-1 level. J Gastroenterol, 1998. 33: p. 447-53

58. Chapman, M.H., Sandanayake, N.S., Andreola, F., et al., Circulating CYFRA 21-1 is a Specific Diagnostic and Prognostic Biomarker in Biliary Tract Cancer. J Clin Exp Hepatol. 1: p. 6-12

59. Lempinen, M., Isoniemi, H., Mäkisalo, H., et al., Enhanced detection of cholangiocarcinoma with serum trypsinogen-2 in patients with severe bile duct strictures. J Hepatol, 2007. 47: p. 677-83

60. Watanabe, H., Enjoji, M., Nakashima, M., et al., Clinical significance of serum RCAS1 levels detected by monoclonal antibody 22-1-1 in patients with cholangiocellular carcinoma. J Hepatol, 2003. 39: p. 559-63

61. Enjoji, M., Yamaguchi, K., Nakamuta, M., et al., Movement of a novel serum tumour marker, RCAS1, in patients with biliary diseases. Dig Liver Dis, 2004. 36: p. 622-7

62. Enjoji, M., Nakamuta, M., Yamaguchi, K., et al., RCAS1, a useful serum marker to predict the recurrence of cancer: two cases of cholangiocarcinoma and pancreatic cancer. Dig Dis Sci, 2004. 49: p. 1654-6 
63. Shi, R.Y., Yang, X.R., Shen, Q.J., et al., High expression of Dickkopf-related protein 1 is related to lymphatic metastasis and indicates poor prognosis in intrahepatic cholangiocarcinoma patients after surgery. Cancer. 119: p. 9931003

64. Tolek, A., Wongkham, C., Proungvitaya, S., et al., Serum alphalbetaglycoprotein and afamin ratio as potential diagnostic and prognostic markers in cholangiocarcinoma. Exp Biol Med (Maywood). 237: p. 1142-9

65. Liu, L., Wang, J., Liu, B., et al., Serum levels of variants of transthyretin down-regulation in cholangiocarcinoma. J Cell Biochem, 2008. 104: p. 74555

66. Alvaro, D., Macarri, G., Mancino, M.G., et al., Serum and biliary insulin-like growth factor I and vascular endothelial growth factor in determining the cause of obstructive cholestasis. Ann Intern Med, 2007. 147: p. 451-9

67. Li, Y.G. and N. Zhang, Clinical significance of serum tumour M2-PK and CA19-9 detection in the diagnosis of cholangiocarcinoma. Dig Liver Dis, 2009. 41: p. $605-8$

68. Jamnongkan, W., Techasen, A., Thanan, R., et al., Oxidized alpha-1 antitrypsin as a predictive risk marker of opisthorchiasis-associated cholangiocarcinoma. Tumour Biol. 34: p. 695-704.

69. Huang, Q., Liu, L., Lui, C.H., et al., Expression of Smad7 in cholangiocarcinoma: prognostic significance and implications for tumor metastasis. Asian Pac J Cancer Prev. 13: p. 5161-5.

70. Alvaro, D., Barbaro, B., Franchitto, A., et al., Estrogens and insulin-like growth factor 1 modulate neoplastic cell growth in human cholangiocarcinoma. Am J Pathol, 2006. 169: p. 877-88

71. Chen, C,Y., Tsai, W.L., Wu, H.C., et al., Diagnostic role of biliary pancreatic elastase for cholangiocarcinoma in patients with cholestasis. Clin Chim Acta, 2008. 390(: p. 82-9

72. TuAyaru, L., Stoeber, K., Webster, G.J., et al., Diagnosis of pancreaticobiliary malignancy by detection of minichromosome maintenance protein 5 in bile aspirates. Br J Cancer, 2008. 98: p. 1548-54

The authors analysed 30 tissue specimens from patients with malignant/benign biliary strictures to study $\mathrm{Mcm} 2$ and -5 expression by immunohistochemistry. Bile samples were also collected prospectively at endoscopic retrograde cholangiopancreatography from 102 consecutive patients with biliary strictures of established $(n=42)$ or indeterminate aetiology $(n=60)$. Minichromosome maintenance protein 5 levels in bile were significantly more sensitive than brush cytology ( 66 vs $20 \% ; \mathrm{P}=0.004$ ) for the detection of malignancy in patients with an indeterminate stricture, with a comparable positive predictive value ( 97 vs $100 \% ; \mathrm{P}=\mathrm{ns}$ ).

73. Chen, C.Y., Lin, X.Z., Tsao, H.C., et al., The value of biliary fibronectin for diagnosis of cholangiocarcinoma. Hepatogastroenterology, 2003. 50: p. 924-7

74. पShen, J., Wang, W., Wu, J., et al., Comparative proteomic profiling of human bile reveals SSP411 as a novel biomarker of cholangiocarcinoma. PLoS One. 7: p. e47476

The authors identified 97 differentially expressed protein spots, corresponding to 49 different genes, of which 38 were upregulated in bile from CCa patients. Western blotting confirmed that phosphoglycerate mutase 1 (brain) (PGAM-1), protein disulfide isomerase family A, member 3 (PDIA3), heat shock $60 \mathrm{kDa}$ protein 1 (chaperonin) (HSPD1) and SSP411 were significantly upregulated in individual bile samples from CC patients. Immunohistochemistry demonstrated these proteins were 
also overexpressed in CCa, relative to normal tissues. SSP411 displayed value as a potential serum diagnostic biomarker for $\mathrm{CC}$, with a sensitivity of $90.0 \%$ and specificity of $83.3 \%$ at a cutoff value of 0.63 .

75. UDhar, D.K., Olde Damink, S.W., Brindley, J.H., et al., Pyruvate kinase M2 is a novel diagnostic marker and predicts tumor progression in human biliary tract cancer. Cancer. 119: p. 575-85

Plasma and bile M2-PK concentrations were measured by enzyme-linked immunosorbent assay in 88 patients with BTC, 79 with benign biliary diseases, and 17 healthy controls. Sensitivity $(90.3 \%)$ and specificity $(84.3 \%)$ of bile M2-PK for malignancy were significantly higher than those for plasma M2-PK and serum carbohydrate antigen 19-9. Transfection of M2-PK in a negatively expressed cell line (HuCCT-1 cells) increased cell invasion, whereas silencing in an M2-PK-positive cell line (TFK cells) decreased tumor nodule formation and cellular invasion.

76. Alpini, G., Invernizzi, P., Gaudio, E., et al., Serotonin metabolism is dysregulated in cholangiocarcinoma, which has implications for tumor growth. Cancer Res, 2008. 68(22): p. 9184-93.

77. Coufal, M., Invernizzi, P., Gaudio, E., et al., Increased local dopamine secretion has growth-promoting effects in cholangiocarcinoma. Int J Cancer. 126: p. 2112-22.

78. Huang, L., Frampton, G., Rao, A., et al., Monoamine oxidase A expression is suppressed in human cholangiocarcinoma via coordinated epigenetic and IL6-driven events. Lab Invest. 92: p. 1451-60

79. Frampton, G., Invernizzi, P., Bernuzzi F., et al., Interleukin-6-driven progranulin expression increases cholangiocarcinoma growth by an Aktdependent mechanism. Gut. 61: p. 268-77

80. $\quad$ पMetzger, J., Negm, A.A., Plentz, R.R., et al., Urine proteomic analysis differentiates cholangiocarcinoma from primary sclerosing cholangitis and other benign biliary disorders. Gut, 2012

The authors evaluated urine proteomic analysis for non-invasive $\mathrm{CCa}$ diagnosis, utilising capillary electrophoresis mass spectrometry to establish a CCa-specific peptide marker model based on the distribution of 42 peptides in $14 \mathrm{CC}, 13 \mathrm{PSC}$ and 14 benign biliary disorder (BBD) patients. In cross-sectional validation of 123 patients, the urine peptide marker model correctly classified 35 of $42 \mathrm{CC}$ patients and 64 of 81 PSC and BBD patients with an area under the curve value of 0.87 (95\% CI 0.80 to $0.92, p=0.0001,83 \%$ sensitivity, $79 \%$ specificity). Evaluation of 101 normal controls resulted in $86 \%$ specificity. All 10 patients with CCa on top of PSC were correctly classified. The majority of sequence-identified peptides are fragments of interstitial collagens with some of them also detected in blood indicating their extrarenal origin.

81. Vasilieva, L.E., S.I. Papadhimitriou, and S.P. Dourakis, Modern diagnostic approaches to cholangiocarcinoma. Hepatobiliary Pancreat Dis Int. 11: p. 349-59

82. Moreno Luna, L.E., Kipp, B., Halling, K.C., et al., Advanced cytologic techniques for the detection of malignant pancreatobiliary strictures. Gastroenterology, 2006. 131: p. 1064-72

83. Bergquist, A., Tribukait, B., Glaumann, H., et al., Can DNA cytometry be used for evaluation of malignancy and premalignancy in bile duct strictures in primary sclerosing cholangitis? J Hepatol, 2000. 33: p. 873-7 
84. Bangarulingam, S.Y., Bjornsson, E., Enders, F., et al., Long-term outcomes of positive fluorescence in situ hybridization tests in primary sclerosing cholangitis. Hepatology. 51: p. 174-80

85. Lindberg, B., Enochsson, L., Tribukait, B., et al., Diagnostic and prognostic implications of DNA ploidy and S-phase evaluation in the assessment of malignancy in biliary strictures. Endoscopy, 2006. 38: p. 561-5

86. ASGE Guidelines: The role of endoscopy in the evaluation and treatment of patients with biliary neoplasia. Gastrointestinal Endoscopy, 2013. 77: p. 167174

87. Ghatnekar, O., Andersson, R., Svensson, M., et al., Modelling the benefits of early diagnosis of pancreatic cancer using a biomarker signature. Int $\mathrm{J}$ Cancer, 2013

88. Briggs, C.D., Neal, C.P., Mann, C.D., et al., Prognostic molecular markers in cholangiocarcinoma: a systematic review. Eur J Cancer, 2009. 45: p. 33-47

89. Wise, C., Pilanthananond, M., Perry, B.F., et al., Mechanisms of biliary carcinogenesis and growth. World J Gastroenterol, 2008. 14: p. 2986-9

90. Boonjaraspinyo, S., Boonmars, T., Wu, Z., et al., Platelet-derived growth factor may be a potential diagnostic and prognostic marker for cholangiocarcinoma. Tumour Biol. 33: p. 1785-802

91. Karamitopoulou, E., Tornillo, L., Zlobec, I., et al., Clinical significance of cell cycle- and apoptosis-related markers in biliary tract cancer: a tissue microarray-based approach revealing a distinctive immunophenotype for intrahepatic and extrahepatic cholangiocarcinomas. Am J Clin Pathol, 2008. 130: p. $780-6$

92. Yoshida, S., Todoroki, T., Ichikawa, Y., et al., Mutations of p16Ink4/CDKN2 and p15Ink4B/MTS2 genes in biliary tract cancers. Cancer Res, 1995. 55: p. 2756-60

93. DeHaan, R.D., Kipp, B.R., Smyrk, T.C., et al., An assessment of chromosomal alterations detected by fluorescence in situ hybridization and pl 6 expression in sporadic and primary sclerosing cholangitis-associated cholangiocarcinomas. Hum Pathol, 2007. 38: p. 491-9

94. Uhm, K.O., Park, Y.N., Lee, J.Y., et al., Chromosomal imbalances in Korean intrahepatic cholangiocarcinoma by comparative genomic hybridization. Cancer Genet Cytogenet, 2005. 157: p. 37-41

95. Ryu, H.S., Chung, J.H., Lee, K., et al., Overexpression of epithelialmesenchymal transition-related markers according to cell dedifferentiation: clinical implications as an independent predictor of poor prognosis in cholangiocarcinoma. Hum Pathol. 43: p. 2360-70

96. Lee, H.J., Chung, J.Y., Hewitt, S.M., et al., HER3 overexpression is a prognostic indicator of extrahepatic cholangiocarcinoma. Virchows Arch. 461: p. 521-30

97. Nutthasirikul, N., Limpaiboon, T., Leelayuwat, C., et al., Ratio disruption of the 133p53 and TAp53 isoform equilibrium correlates with poor clinical outcome in intrahepatic cholangiocarcinoma. Int J Oncol. 42: p. 1181-8

98. Koo, S.H., Ihm, C.H., Kwon, K.C., et al., Genetic alterations in hepatocellular carcinoma and intrahepatic cholangiocarcinoma. Cancer Genet Cytogenet, 2001. 130: p. 22-8

99. Shiraishi, K., Okita, K., Harada, T., et al., Comparative genomic hybridization analysis of genetic aberrations associated with development and progression of biliary tract carcinomas. Cancer, 2001. 91: p. 570-7 
100. Andresen, K., Boberg, K.M., Vedeld, H.M., et al., Novel target genes and a valid biomarker panel identified for cholangiocarcinoma. Epigenetics. 7: p. 1249-57

101. Bartel, D.P., MicroRNAs: target recognition and regulatory functions. Cell, 2009. 136: p. 215-33

102. Garzon, R., G. Marcucci, and C.M. Croce, Targeting microRNAs in cancer: rationale, strategies and challenges. Nat Rev Drug Discov. 9: p. 775-89

103. Meng, F., Wehbe-Janek, H., Henson, R., et al., Epigenetic regulation of microRNA-370 by interleukin-6 in malignant human cholangiocytes. Oncogene, 2008. 27: p. 378-86

104. Braconi, C., N. Huang, and T. Patel, MicroRNA-dependent regulation of DNA methyltransferase-1 and tumor suppressor gene expression by interleukin-6 in human malignant cholangiocytes. Hepatology. 51: p. 881-90

105. Zhong, X.Y., Yu, J.H., Zhang, W.G., et al., MicroRNA-421 functions as an oncogenic miRNA in biliary tract cancer through down-regulating farnesoid $X$ receptor expression. Gene. 493: p. 44-51

106. Kemper, J.K., Regulation of FXR transcriptional activity in health and disease: Emerging roles of FXR cofactors and post-translational modifications. Biochim Biophys Acta. 1812: p. 842-50

107. Teodoro, J.S., A.P. Rolo, and C.M. Palmeira, Hepatic FXR: key regulator of whole-body energy metabolism. Trends Endocrinol Metab. 22: p. 458-66

108. Zhang, J., C. Han, and T. Wu, MicroRNA-26a promotes cholangiocarcinoma growth by activating beta-catenin. Gastroenterology. 143: p. 246-56 e8

109. He, Q., Cai, L., Shuai, L., et al., Ars2 is overexpressed in human cholangiocarcinomas and its depletion increases PTEN and PDCD4 by decreasing microRNA-21. Mol Carcinog. 52: p. 286-96

110. Razumilava, N., Bronk, S.F., Smoot, R.L., et al., miR-25 targets TNF-related apoptosis inducing ligand (TRAIL) death receptor-4 and promotes apoptosis resistance in cholangiocarcinoma. Hepatology. 55: p. 465-75

111. Stutes, M., S. Tran, and S. DeMorrow, Genetic and epigenetic changes associated with cholangiocarcinoma: from DNA methylation to microRNAs. World J Gastroenterol, 2007. 13: p. 6465-9

112. Mott, J.L., Kobayashi, S., Bronk, S.F., et al., mir-29 regulates Mcl-1 protein expression and apoptosis. Oncogene, 2007. 26: p. 6133-40

113. Meng, F., Henson, R., Lang, M., et al., Involvement of human micro-RNA in growth and response to chemotherapy in human cholangiocarcinoma cell lines. Gastroenterology, 2006. 130: p. 2113-29

114. Murugaesu, N., S.K. Chew, and C. Swanton, Adapting clinical paradigms to the challenges of cancer clonal evolution. Am J Pathol. 182: p. 1962-71 


\begin{tabular}{|c|c|c|c|c|c|c|c|}
\hline \multirow[b]{2}{*}{ Biomarker/Combination } & \multirow[b]{2}{*}{$\begin{array}{c}\text { Sample } \\
\text { Type }\end{array}$} & \multicolumn{3}{|c|}{ No. Samples } & \multicolumn{2}{|c|}{ Performance } & \multirow[b]{2}{*}{ Reference } \\
\hline & & BTC & $\begin{array}{l}\text { Benign/ } \\
\text { Cancer } \\
\text { Controls }\end{array}$ & $\begin{array}{l}\text { Healthy } \\
\text { Controls }\end{array}$ & Sensitivity & Specificity & \\
\hline IL6 & Serum & 26 & 26 & 23 & $73 \%$ & $92 \%$ & {$[46]$} \\
\hline IL-6 & Serum & 45 & 40 & 10 & $71 \%$ & $90 \%$ & [47] \\
\hline IL6/LRG1/CA19-9 & Serum & 31 & 13 & - & \multicolumn{2}{|c|}{ AUC 0.98} & [48] \\
\hline MUC5AC & Serum & 179 & 122 & 74 & $62.6 \%$ & $96.9 \%$ & {$[50]$} \\
\hline MUC5AC & Serum & 169 & 60 & 30 & $71 \%$ & $90 \%$ & [49] \\
\hline MUC5AC & Serum & 39 & 33 & - & $44 \%$ & $96 \%$ & {$[51]$} \\
\hline CYFRA21-1 & Serum & 55 & 90 & - & $74.7 \%$ & $92.2 \%$ & {$[56]$} \\
\hline CYFRA21-1 & Serum & 66 & 58 & - & $56 \%$ & $88 \%$ & {$[58]$} \\
\hline CYFRA21-1/CA19-9 & Serum & 66 & 58 & - & $45 \%$ & $96 \%$ & {$[58]$} \\
\hline PRSS2 & Serum & 38 & 46 & - & \multicolumn{2}{|c|}{ AUC 0.804} & [59] \\
\hline RCAS1 & Serum & 23 & 72 & 35 & $73.9 \%$ & $96.2 \%$ & {$[60]$} \\
\hline RCAS1 & Serum & 31 & 107 & - & $74.4 \%$ & $91.0 \%$ & {$[61]$} \\
\hline A1BG/AFM ratio & Serum & 64 & 4 & 20 & $84.4 \%$ & $87.5 \%$ & {$[64]$} \\
\hline TTR/CA19-9 & Serum & 56 & 322 & 53 & $98.2 \%$ & $100 \%$ & [65] \\
\hline VEGF & Serum & 29 & 44 & - & \multicolumn{2}{|c|}{ AUC 0.744} & [66] \\
\hline M2-PK & Serum & 115 & 85 & 120 & $84.2 \%$ & $90.0 \%$ & [67] \\
\hline МАС-2BP/CA19-9 & Bile & 26 & 52 & 0 & \multicolumn{2}{|c|}{ AUC 0.75} & {$[42]$} \\
\hline IGF1 & Bile & 29 & 44 & - & \multicolumn{2}{|c|}{ AUC 1.000} & {$[66]$} \\
\hline Elastase & Bile & 22 & 28 & - & $82 \%$ & $89 \%$ & [71] \\
\hline MCM5 & Bile & 27 & 75 & - & $66 \%$ & N/A & {$[72]$} \\
\hline Fibronectin & Bile & 28 & 67 & - & $57 \%$ & $79 \%$ & [73] \\
\hline SPATA20 & Bile & 35 & 23 & 47 & $90 \%$ & $83.3 \%$ & {$[74]$} \\
\hline M2-PK & Bile & 88 & 79 & 17 & $90.3 \%$ & $84.3 \%$ & [75] \\
\hline 42 Peptides & Urine & 42 & 81 & - & $83 \%$ & $79 \%$ & {$[80]$} \\
\hline
\end{tabular}

Table 1. A summary of recent novel biomarker studies for the diagnosis and assessment of patients with BTC (BTC-Biliary tract cancer; AUC- Area under ROC curve; MAC-2BP- MAC-2 binding protein; CA19-9- Carbohydrate antigen 19-9; IL6- Interleukin-6; LRG1; MUC5AC- Mucin5AC; CYFRA21-1- Soluble fragment of cytokeratin 19; PRSS2- Trypsinogen-2; TTR- Transthyretin; RCAS1- Receptor binding cancer antigen 1; A1BG- $\alpha 1 \beta$-Glycoprotein; AFM- Afamin; VEGF- Vascular endothelial growth factor; IGF1- Insulin-like Growth Factor-1; MCM5- Mini chromosome maintenance protein 5; SPATA20- Spermatogenesis-associated protein 20; M2PK- M2 isotope of pyruvate kinase). 